\title{
Magnetism of Molecular Conductors
}

\author{
Manuel Almeida \\ C2TN-Centro de Ciências e Tecnologias Nucleares, Instituto Superior Técnico, Universidade de Lisboa, \\ P-2695-066 Bobadela LRS, Portugal; malmeida@ctn.tecnico.ulisboa.pt; Tel.: +351-219-946-171
}

Received: 19 June 2017; Accepted: 21 June 2017; Published: 27 June 2017

The study of the magnetic properties of molecular conductors has experienced, during the last decades, a very significant evolution, comprising systems of increasing molecular complexity and moving towards multifunctional materials, namely by their incorporation in conducting networks of different paramagnetic centers. In this context, molecular magnetic conductors have emerged at the intersection between the fields of molecule-based conductors and molecule-based magnets as a very exciting class of multifunctional materials in which the interaction and synergy between conduction electrons and localized magnetic moments can lead to new phenomena, complex phase diagrams, and different ground states, with a large potential for technological applications, namely in electronic devices, sensors and in spintronics. Among these phenomena are unusual field-induced transitions, including magnetic field-induced superconductivity, very large magnetoresistance effects, conductors that are switchable by magnetic field, changes of magnetic ordering or spin state, etc.

This Special Issue of Magnetochemistry features a collection of research contributions illustrating recent achievements in different aspects of this topic concerning the development, study and understanding of the magnetic properties of molecular conductors and their applications. Quite different types of compounds are considered.

A contribution by Tamotsu Inabe et al. [1], reviews a series of compounds based on axially ligated phthalocyanines. Metal phthalocyanines are one of the first examples of compounds where, in addition to delocalized $\pi$-conduction electrons in the ligand, there can also be localized magnetic moments for some metals. The $\pi-d$ interaction of these local moments embedded in the sea of conduction electrons has long since been identified as a source of possible interesting phenomena. In this contribution, the properties of $\operatorname{TPP}\left[\mathrm{M}(\mathrm{Pc})(\mathrm{CN})_{2}\right]_{2}$ compounds (TPP = tetraphenylphosphonium, $\mathrm{Pc}=$ phthalocyaninato), with $\mathrm{M}=\mathrm{Fe}$ and $\mathrm{Cr}$ are reviewed, emphasizing carrier localization and charge disproportionation enhanced by the interaction between local magnetic moments and conduction $\pi$-electrons ( $\pi-\mathrm{d}$ interaction), and the large negative magnetoresistance, reflecting the difference in the anisotropy of different $\mathrm{d}-\mathrm{d}, \pi-\mathrm{d}$, and $\pi-\pi$ interactions.

Two other contributions in this issue concern a family of compounds with two types of chains (conducting and magnetic), based on the organic perylene donor and inorganic $\left[\mathrm{M}(\mathrm{mnt})_{2}\right]$ anions, which have been studied for more than 30 years, but are still unique among molecular materials. In a review by Jean-Paul Pouget et al. [2], the structural instabilities exhibited of these salts are reviewed and discussed in relation to the magnetic properties of the 1D spin-Peierls (SP) instability of the dithiolate stacks, showing, in particular, that $\alpha$-(Per $)_{2}\left[\mathrm{M}(\mathrm{mnt})_{2}\right]$ salts exhibit the physical properties expected of a two-chain Kondo lattice. In another contribution by Manuel Matos et al. [3], these compounds are also addressed, the properties of the solid solutions (Per $)_{2}\left[\mathrm{Pt}_{x} \mathrm{Au}_{(1-x)}(\mathrm{mnt})_{2}\right]$ being described, probing the incorporation of paramagnetic $\left[\mathrm{Pt}(\mathrm{mnt})_{2}\right]$ impurities in diamagnetic chains, and the effect of breaking paramagnetic chains with diamagnetic centers.

Another contribution by Yugo Oshima et al. [4] concerns $\lambda$-(BETS $)_{2} \mathrm{FeCl}_{4},(\mathrm{BETS}=$ bis (ethylenedithio)tetraselenafulvalene), a very relevant compound in the context of the topic of this special issue, due to the magnetic field-induced superconducting state observed. Studies on the antiferromagnetic insulating phase of this compound are reviewed, and new ESR data on the solid 
solutions with diamagnetic anions, $\lambda$-(BETS) ${ }_{2} \mathrm{Fe}_{x} \mathrm{Ga}_{1-x} \mathrm{Cl}_{4}$, are provided, showing that there is no sign of paramagnetic Fe spins in the antiferromagnetic ground state, which has been a point of previous debate.

Another contribution by Maria Laura Mercuri [5] reviews Anilato-Based Molecular Materials, illustrating the large potential of anilato ligands, derivatives of the 2,5-dioxy-1,4-benzoquinone framework with various substituents $(\mathrm{X}=\mathrm{H}, \mathrm{Cl}, \mathrm{Br}, \mathrm{I}, \mathrm{CN}$, etc.) in different positions as molecular building blocks for the design of a rich variety of materials with peculiar magnetic and/or conducting properties.

Molecular radical units with unpaired electrons have been the basis of one of the earliest devised strategies for achieving organic conductors. In the contribution of Manuel Souto et al. [6], a new molecular dyad is reported, based on a monopyrrolo-tetrathiafulvalene electron donor linked by a $\pi$-conjugated bridge to a perchlorotriphenylmethyl radical, with interesting properties and the potential to give rise to new radical conductors in the solid state. The combination of radical units with electroactive donor networks is another strategy for preparing magnetic conductors, and in this issue Kazuki Horikiri and Hideki Fujiwara describe charge transfer salts of a new EDT-TTF (ethylenedithiotetrathiafulvalene) donor containing a radical through a $\pi$-conjugated vinylene spacer, with diamagnetic $\mathrm{GaCl}_{4}$ and paramagnetic $\mathrm{FeCl}_{4}$ anions with strong $\pi-\mathrm{d}$ interactions [7]. Another contribution by Hiroki Akutsu et al. describes two dmit-based salts with a stable organic radical-substituted ammonium cation, exhibiting magnetic contributions from segregated 2D anionic and cationic sub-lattices [8].

BEDT-TTF (Bis(ethylenedithio)tetrathiafulvalene or ET) is one of the most successful electron donors, being the basis of a very large number of charge transfer salts with metallic and superconducting properties. In the contribution of Samia Benmansour et al. [9], two novel paramagnetic conductors of this donor-as salts with oxalate anionic layers containing high spin $\mathrm{Mn}(\mathrm{III})(\mathrm{S}=2)$ and $\mathrm{Mn}(\mathrm{II})(\mathrm{S}=5 / 2)$ ions-are described. BEDT-TTF charge transfer salts are characterized by a very large structural diversity associated with different electrical and magnetic properties. Tadashi Kawamoto et al., in their contribution to this issue [10], describe structural, transport, and magnetic properties two $\delta$-type polymorphs of the salt (BEDT-TTF) ${ }_{2} \mathrm{TaF}_{6}$ with Charge Ordering transitions.

The exploration of interaction effects between delocalized conduction electrons and paramagnetic centers by incorporation of spin-crossover units in conducting networks remains a challenge in molecular multifunctional conducting and magnetic materials. As an example of current efforts under such strategy, a contribution by Yuri N. Shvachko et al. [11] describes three conducting systems based on the electron acceptor TCNQ and spin cross-over Fe(III) cations, displaying strong interactions between local magnetic moments of Fe(III) ions and electron spins of the organic TCNQ network.

Finally, Royama Yamamoto et al. [12] explore the incorporation of photosensitive dyes to optically control and trigger conduction and magnetism in the photo-excited states of organic materials. In their contribution, these authors report a new type of salt based on [Ni(dmit $\left.)_{2}\right]$ (dmit = 1,3-dithiole-2-thione-4,5-dithiolate and 3,3'-Dihexyloxacarbocyanine monocation, exhibiting photoconductivity with photo-excited spins, demonstrating the possibility of preparing paramagnetic organic photo responsive semiconductors.

From the large diversity of compounds considered in this special issue, illustrating current strategies for the development of molecular magnetic conductors, it becomes clear that the magnetic conductors are a topic of increasing interest among molecular materials, where several significant developments and relevant applications are expected in the near future.

Conflicts of Interest: The authors declare no conflict of interest. 


\section{References}

1. Inabe, T.; Hanasaki, N. Axially Ligated Phthalocyanine Conductors with Magnetic Moments. Magnetochemistry 2017, 3, 18. [CrossRef]

2. Pouget, J.-P.; Foury-Leylekian, P.; Almeida, M. Peierls and Spin-Peierls Instabilities in the $\operatorname{Per}_{2}\left[\mathrm{M}(\mathrm{mnt})_{2}\right]$ Series of One-Dimensional Organic Conductors; Experimental Realization of a 1D Kondo Lattice for M $=\mathrm{Pd}$, $\mathrm{Ni}$ and Pt. Magnetochemistry 2017, 3, 13. [CrossRef]

3. Matos, M.; Bonfait, G.; Santos, I.C.; Afonso, M.L.; Henriques, R.T.; Almeida, M. The solid solutions $(\mathrm{Per})_{2}\left[\mathrm{Pt}_{x} \mathrm{Au}_{(1-x)}(\mathrm{mnt})_{2}\right]$; Alloying para- and diamagnetic anions in two-chain compounds. Magnetochemistry 2017, 3, 22. [CrossRef]

4. Oshima, Y.; Cui, H.-B.; Kato, R. Antiferromagnetic Insulating Ground State of Molecular $\pi-\mathrm{d}$ System $\lambda$-(BETS) ${ }_{2} \mathrm{FeCl}_{4}$ (BETS = Bis(ethylenedithio)tetraselenafulvalene): A Theoretical and Experimental Review. Magnetochemistry 2017, 3, 10. [CrossRef]

5. Mercuri, M.L.; Congiu, F.; Concas, G.; Sahadevan, S.A. Recent Advances on Anilato-Based Molecular Materials with Magnetic and/or Conducting Properties. Magnetochemistry 2017, 3, 17. [CrossRef]

6. Souto, M.; Bendixen, D.; Jensen, M.; Díez-Cabanes, V.; Cornil, V.J.; Jeppesen, J.O.; Ratera, I.; Rovira, C.; Veciana, J. Synthesis and Characterization of Ethylenedithio-MPTTF-PTM Radical Dyad as a Potential Neutral Radical Conductor. Magnetochemistry 2016, 2, 46. [CrossRef]

7. Horikiri, K.; Fujiwara, H. New Ethylenedithio-TTF Containing a 2,2,5,5-Tetramethylpyrrolin-1-yloxyl Radical through a Vinylene Spacer and Its $\mathrm{FeCl}_{4}{ }^{-}$Salt—Synthesis, Physical Properties and Crystal Structure Analyses. Magnetochemistry 2017, 3, 8. [CrossRef]

8. Akutsu, H.; Turner, S.S.; Nakazawa, Y. New Dmit-Based Organic Magnetic Conductors $\left(\mathrm{PO}-\mathrm{CONH}-\mathrm{C}_{2} \mathrm{H}_{4} \mathrm{~N}\left(\mathrm{CH}_{3}\right)_{3}\right)\left[\mathrm{M}(\mathrm{dmit})_{2}\right]_{2}(\mathrm{M}=\mathrm{Ni}, \mathrm{Pd})$ Including an Organic Cation Derived from a 2,2,5,5-Tetramethyl-3-pyrrolin-1-oxyl (PO) Radical. Magnetochemistry 2017, 3, 11. [CrossRef]

9. Benmansour, S.; Sánchez-Máñez, Y.; Gómez-García, C.J. Mn-Containing Paramagnetic Conductors with Bis(ethylenedithio)tetrathiafulvalene (BEDT-TTF). Magnetochemistry 2017, 3, 7. [CrossRef]

10. Kawamoto, T.; Kurata, K.; Mori, T.; Kumai, R. Charge Ordering Transitions of the New Organic Conductors $\delta_{\mathrm{m}}$ - and $\delta_{\mathrm{o}}-(\mathrm{BEDT}-\mathrm{TTF})_{2} \mathrm{TaF}_{6}$. Magnetochemistry 2017, 3, 14. [CrossRef]

11. Shvachko, Y.N.; Starichenko, D.V.; Korolyov, A.V.; Kotov, A.I.; Buravov, L.I.; Zverev, V.N.; Simonov, S.V.; Zorina, L.V.; Yagubskii, E.B. The Highly Conducting Spin-Crossover Compound Combining Fe(III) Cation Complex with TCNQ in a Fractional Reduction State. Synthesis, Structure, Electric and Magnetic Properties. Magnetochemistry 2017, 3, 9. [CrossRef]

12. Yamamoto, R.; Yamamoto, T.; Ohara, K.; Naito, T. Dye-Sensitized Molecular Charge Transfer Complexes: Magnetic and Conduction Properties in the Photoexcited States of $\mathrm{Ni}(\mathrm{dmit})_{2}$ Salts Containing Photosensitive Dyes. Magnetochemistry 2017, 3, 20. [CrossRef] 